\title{
Shifting of the resonance location for planets embedded in circumstellar disks
}

\begin{abstract}
F. Marzari
Dipartimento di Fisica, University of Padova, Via Marzolo 8, 35131 Padova, Italy

e-mail: Francesco.Marzari@pd.infn.it

Received 10 October 2017 / Accepted 3 December 2017

ABSTRACT

Context. In the early evolution of a planetary system, a pair of planets may be captured in a mean motion resonance while still embedded in their nesting circumstellar disk.

Aims. The goal is to estimate the direction and amount of shift in the semimajor axis of the resonance location due to the disk gravity as a function of the gas density and mass of the planets. The stability of the resonance lock when the disk dissipates is also tested. Methods. The orbital evolution of a large number of systems is numerically integrated within a three-body problem in which the disk potential is computed as a series of expansion. This is a good approximation, at least over a limited amount of time.

Results. Two different resonances are studied: the 2:1 and the 3:2. In both cases the shift is inwards, even if by a different amount, when the planets are massive and carve a gap in the disk. For super-Earths, the shift is instead outwards. Different disk densities, $\Sigma$, are considered and the resonance shift depends almost linearly on $\Sigma$. The gas dissipation leads to destabilization of a significant number of resonant systems, in particular if it is fast.

Conclusions. The presence of a massive circumstellar disk may significantly affect the resonant behavior of a pair of planets by shifting the resonant location and by decreasing the size of the stability region. The disk dissipation may explain some systems found close to a resonance but not locked in it.
\end{abstract}

Key words. planet-disk interactions

\section{Introduction}

During the early phases of evolution of a planetary system it may happen that two planets are trapped in a mean-motion resonance. By inspecting known multi-planet systems, a significant number of planets can be found very close to a commensurability and, in particular, the closeness to low-order resonances such as 2:1 and 3:2 is frequently observed (Wright et al. 2011; Fabrycky \& Kepler Science Team 2012). For example, the 3:2 resonance is possibly present in HD 45364 (Rein et al. 2010), KOI 55 (Kepler 70) (Charpinet et al. 2011), and many other Kepler systems (Steffen et al. 2013). Well-known systems with planets suspected to be in the 2:1 resonance are Gliese 876 and HD 82943.

The assembly of a resonant configuration may be the result of formation or dynamical evolution of the system. Multi-planet systems may directly form in a chain of resonances like Kepler223 (Kepler-223's two innermost planets are in a 4:3 resonance, the second and third are in a 3:2 resonance and, finally, the third and fourth are in a 4:3 resonance) leading to a tightly packed orbital configuration (Mills et al. 2016). Direct in situ formation in a dense disk (Hansen \& Murray 2013) or inside-out formation (Chatterjee \& Tan 2014) may lead to resonant pairs even if the lock may be disrupted later on during the evolution of the system.

A mechanism often invoked to explain resonant configurations is convergent migration where two planets are moved inward together by tidal interaction with the circumstellar disk (e.g., Masset \& Snellgrove 2001; Lee \& Peale 2002; Moorhead \& Adams 2005; Thommes 2005; Beaugé et al. 2006; Crida et al. 2008). In a typical planet migration scenario, a giant planet drifting inwards is reached from outside by a less massive planet migrating at a faster pace. Depending on the disk density, the couple can be trapped in different mean motion resonances (D’Angelo \& Marzari 2012), in particular the 2:1 or the 3:2. This may lead to a period of coupled inward/outward migration until the disk dissipates. The "grand tack" scenario (Walsh et al. 2011) is a possible application of this mechanism. It is also possible that the inner giant planet triggers the formation of a tightly packed planetary system with all the members in resonance.

Once trapped in resonance, the common gap determines the coupled migration rate (D'Angelo \& Marzari 2012). However, even a different dynamical effect comes into play when the planets are in resonance. In addition to the apsidal precession rate induced by the resonant perturbations, there is a significant contribution forced by the overall gravitational force of the gas disk. This can significantly affect the dynamics of the planets in resonance by shifting the location of potential commensurabilities between them (Tamayo et al. 2015) and, during the disk dissipation, may also lead to resonance escape.

Various analytical approaches have been developed to compute this precession rate. The initial physical assumptions at the basis of all these models are equivalent, but they gradually depart from one another in the subsequent way of manipulating the equations and in the approximations adopted to derive a simple handy expression (Mestel 1963; Ward 1981; Binney \& Tremaine 2008; Silsbee \& Rafikov 2015; Fontana \& Marzari 2016). However, these formalisms have been applied in most cases to compute the circulation rate of a single planet, while in the case of two planets in resonance, the situation is more complex. In the majority of resonant configurations an apsidal 
libration is also present and the change in the secular circulation frequency due to the disk potential affects the system of the two planets as a whole, not each body individually. Therefore it is not easy to predict the resonance shift because it is due to the interaction between the secular circulation frequency at the resonance and the circulation forced by the disk potential on each planet. For this reason a numerical approach is adopted here where the orbits of the planets are computed with the addition of the gravitational force of the disk acting on each planet. The formalism developed by Ward (1981) is used and the series expansions developed to compute the gravitational potential of the disk are numerically computed at a high order. The shift in the resonance location due to the disk gravity is computed for different values of the disk density and planet masses. The parameter space is huge, so here the exploration is limited to a few cases that provide information on the important dynamical processes related to the effects of the disk potential on the planet precession rate. In Fontana \& Marzari (2016) the comparison between the apsidal precession rate predicted by the analytical approach of Ward (1981) has been compared to that computed by hydrodynamical simulations performed with the code FARGO (Masset 2000) for a single planet of different masses. A good agreement has been observed between analytical and numerical results (Fontana \& Marzari 2016) implying that the angular momentum exchange, related to the gas-planet interaction and causing planet migration, does not significantly influence the resonance location which is instead mostly related to the apsidal frequency change due to the disk gravity.

In Sect. 2 the critical arguments of a planetary resonance are described, while in Sect. 3 the analytical formulas used to compute the disk potential are briefly summarized. Section 4 is devoted to the numerical algorithm and to the definition of the initial conditions. The results of the numerical modeling for the 2:1 resonance and for different planetary masses are described in Sects. 5 and 6 is dedicated to the 3:2 resonance. Finally, a discussion of the results and the conclusions are given in Sect. 6 .

\section{Planets in resonance}

The focus of this paper is on first-order mean motion resonances like the 2:1 and 3:2 which are the most frequently encountered in exoplanetary systems. From a computational point of view, the best way to determine when two planets are in resonance is to check the behavior of the two critical angles:

$\sigma_{1}=(j+1) \lambda_{2}-j \lambda_{1}-\varpi_{1}$,

$\sigma_{2}=(j+1) \lambda_{2}-j \lambda_{1}-\varpi_{2}$,

where $\lambda_{i}$ is the mean longitude of planet $i$ while $\varpi_{i}$ is its apsidal longitude. Outside the resonance, both $\sigma_{1}$ and $\sigma_{2}$ circulate from $0^{\circ}$ to $360^{\circ}$. When the planets are in resonance, at least one of the critical arguments is a libration regime and oscillates about either $0^{\circ}$ or $180^{\circ}$ (Beaugé \& Michtchenko 2003; Callegari et al. 2004). When both $\sigma_{1}$ and $\sigma_{2}$ librate, a state of apsidal libration is met where the circulation frequencies of the apsidal longitude of the two planets match.

The easiest way to test if the two planets are in resonance during an N-body numerical simulation is to define two stripes around both $0^{\circ}$ and $180^{\circ}$ with a small width (in all simulations a half-width of $5^{\circ}$ is chosen). If, during the numerical integration, both the critical angles cross these stripes then the system is assumed to be out of resonance. Until this condition is met, the system is assumed to be resonant. This is a brute force method but it is very efficient when handling the outcome of a large number of numerical simulations of potentially resonant systems.

From the above expressions of the critical arguments it can be deduced that the location of the resonance is affected by any change in the apsidal frequencies of the planets due to an external perturbation such as the disk gravity. Since the latter alters the circulation frequency of $\dot{\varpi}_{i}$, then a change in the circulation period of $\lambda_{2}$ is required to restore the resonant lock (the orbit of the inner planet is fixed in all models, so the frequency of $\lambda_{1}$ does not change). Only a new frequency of $\lambda_{2}$, and then a different value of the outer planet semimajor axis, is able to maintain $\sigma_{i}$ in a libration motion. It is noteworthy that the disk gravity affects the inner and outer planet to a different extent and the shift in the frequency of $\lambda_{2}$ of the outer planet indirectly includes the change in the frequency of $\dot{\varpi}_{1}$. This is because the planets are locked in resonance and react to the disk gravity as a single entity.

\section{Computation of the disk potential}

In all models, a thin disk is considered with a surface gas density profile $\Sigma(r)$ parameterized as a power law of the form:

$\Sigma(r)=\Sigma_{0} r^{-p}$.

The disk gravitational potential is computed using the formula of Ward (1981):

$$
\begin{aligned}
U^{W}(r)= & 2 \pi G r \sum(r) \sum_{k=0}^{+\infty} A_{k}\left[\frac{(4 k+1)}{(2 k+2-p)(2 k-1+p)}\right. \\
& -\left(\frac{1}{2 k+2-p}\right)\left(\frac{R_{\text {in }}}{r}\right)^{2 k+2-p} \\
& \left.-\left(\frac{1}{2 k-1+p}\right)\left(\frac{r}{R_{t}}\right)^{2 k-1+p}\right] .
\end{aligned}
$$

In the above equation, $r$ is the radial distance from the star, $R_{\text {in }}$ and $R_{\text {out }}$ are the inner and outer borders of the disk, respectively, and $A_{k}=\left[(2 k) ! / 2^{2 k}(k !)^{2}\right]^{2}$.

If the planets are massive enough, they may open a common gap and a more accurate approach for the computation of the disk potential is given by the following equation (Ward 1981):

$$
\begin{aligned}
U^{W}= & 2 \pi G \Sigma(r) \sum_{k=0}^{+\infty} A_{k}\left[\frac{1}{(2 k+2-p)}\left(\frac{D_{\text {in }}}{r}\right)^{2 k+2-p}\left(1-\left(\frac{R_{\text {in }}}{D_{\text {in }}}\right)^{2 k+2-p}\right)\right. \\
& \left.+\frac{1}{(2 k-1+p)}\left(\frac{r}{D_{\text {out }}}\right)^{2 k-1+p}\left(1-\left(\frac{D_{\text {out }}}{R_{t}}\right)^{2 k-1+p}\right)\right]
\end{aligned}
$$

The inner and outer borders of the gap are $D_{\text {in }}, D_{\text {out }}$, respectively. To properly use this equation we need a rough estimate of the values of both $D_{\text {in }}$ and $D_{\text {out }}$. As an order of magnitude we adopt the equation for the size of the gap carved by a single giant planet given in Isella et al. (2016):

$W \sim C \cdot R_{H}$,

where the constant $C$ can range from 4 to 8 and $R_{H}$ is the Hill's sphere. In the numerical modeling, two different values for $C$ were tested: either 3 or 4 . No significant differences were observed in the outcome, therefore in all the simulations $C=4$ is adopted. In the numerical computation of the disk potential 
we extend the summation of the series expansion up to $k=100$. Some tests with a higher numberof terms show that no significant contributions to the potential come from higher-order terms at the cost of a considerable slowing down of the computations.

\section{The numerical setup}

The parameter space of two planets in resonance is huge, so only a few representative scenarios are considered. Two different combinations of planet masses are considered: first a pair of massive planets where the inner planet has the mass of Jupiter (the inner one, $\left.m_{1}=M_{\mathrm{J}}\right)$ and the outer one that of Saturn $\left(m_{2}=M_{\mathrm{S}}\right)$. This choice of the mass ratio is dictated by the migration-driven resonant trapping mechanism which, to be efficient, requires the outer body to be less massive than the inner one. A second less massive case is contemplated where the mass of the inner planet is set to $m_{1}=5 M_{\mathrm{E}}$ (a super-Earth) while that of the outer one is $m_{2}=3 M_{\mathrm{E}}$. The initial semimajor axis of the inner planet $a_{1}$ is fixed to 5 au for the massive planets case and to 5 and 1 au in the super-Earths case. The semimajor axis of the second planet $a_{2}$ is varied within a wide range around the exact resonant value. The orbits of the planets are assumed to be coplanar to the disk and the eccentricity is randomly sampled between 0 and 0.2 . The orbital angles (mean anomaly, pericenter argument and longitude of node) of the inner planet are set to zero while those of the outer planet are randomly chosen between 0 and $2 \pi$. To define the range within which to look for resonant systems, we use a rough estimate of the resonant width for circular orbits:

$\frac{\Delta n}{n_{R}} \simeq \mu^{2 / 3}$

applicable to first order resonances, where $n_{R}$ is the mean motion at the exact commensurability while $\mu$ is the mass ratio. We estimate from the above equation a value of $\Delta a$ and multiply it by 10 in order to also cover the models where the disk potential shifts inwards or outwards with respect to the exact resonant location.

As a reference value for the gas density, the minimum mass solar nebula (MMSN) is adopted (Chiang \& Youdin 2010):

$\Sigma(r)=2200 \times r^{-3 / 2} \mathrm{~g} \mathrm{~cm}^{-2}$.

According to Chiang \& Laughlin (2013) a minimum-mass extrasolar nebula (MMEN) can be derived from the data of the Kepler mission neglecting significant migration of planets. Their average MMEN is approximately five times more massive than the MMSN and from their data even more massive disks could potentially be found around solar-type stars. Similar results are obtained also by Raymond \& Cossou (2014). Assuming that in any case massive disks can be encountered, in the simulations two different values for $\Sigma_{0}$ are adopted. Either $\Sigma_{0}=\Sigma_{\text {MMSN }}=2200 \mathrm{~g} \mathrm{~cm}^{-2}$ is used or a massive disk is modeled with $\Sigma_{0}=5 \cdot \Sigma_{\text {MMSN }}$. The shift in resonance for intermediate values of $\Sigma_{0}$ can be extrapolated from these cases. The scenario explored here assumes that planets can form very rapidly before significant dissipation of the disk. They are trapped in resonance either by convergent migration or by direct formation in a resonant configuration. Different dynamical paths leading to resonance can be envisaged in particular in multi-planet systems.

When modeling the dynamical evolution of two planets in resonance embedded in a circumstellar disk, the best approach would be to exploit a hydrodynamical code which includes the evolution of the gas. However, this approach would require heavy numerical computations simply for determining the stability of a single system. While searching for the averaged shift in the resonant location, a large number of systems have to be integrated since not all systems that are stable for a given value of the disk density $\Sigma$ are stable also for a different value. Furthermore, it is also interesting to test the potential shrinking (or widening) of the resonance volume in phase space as a function of $\Sigma$. For these reasons a statistical approach is to be preferred where thousands of systems are numerically integrated to evaluate the changes in the resonant structure for different disks. To pursue this objective it is not possible to exploit hydrodynamical simulations and an $\mathrm{N}$-body approach where the disk potential is computed with the equations of Ward (1981) would allow a more accurate sampling of the parameter space. Here we compute a large number of different static resonant configurations where the planets do not migrate. The stability of the resonance lock is investigated over $10^{5} \mathrm{yr}$. It is possible to include in the numerical models a dissipative term simulating the migration of the system but this would render the estimate of the shift in semimajor axis of the planets in resonance more difficult to evaluate. During migration, the secular frequencies will slightly change due to the semimajor axis variation and the contribution of the disk potential will change with time. For this reason, to evaluate the local amount of shift in the resonance location it is better to use a static configuration. In addition, as shown in Fontana \& Marzari (2016), the comparison of the analytical formulas with numerical simulations performed with the hydrodynamical code FARGO (Masset 2000) have shown very good agreement. It is then expected that also in a more complex model the apsidal precession rate is dominated by the disk potential and well modeled by the analytical formalism of Ward (1981).

Once the dependence of the resonant phase space on different values of $\Sigma$ and on different masses and semimajor axes of the planets has been explored, it is interesting to test whether the disk dissipation may destroy the resonant lock. With this goal in mind, the same two-planet systems have been integrated with a model where the disk density $\Sigma$ exponentially decreases with time:

$\Sigma(r, t)=\Sigma_{0} r^{-p} \mathrm{e}^{-t / \tau}$,

where $\tau$ is the dissipation timescale. As a good compromise between the need for fast numerical integrations and reasonable values for the dissipation timescale of protoplanetary disks, $\tau$ has been set to $5 \times 10^{5} \mathrm{yr}$. The number of stable systems at the end of the numerical integrations is then compared to the "static" case to test how often a resonant system can be destabilized by the change in the apsidal frequencies related to the disk dissipation.

\section{The 2:1 resonance}

The first resonance to be studied is the 2:1 which was analyzed for the first time by Lee \& Peale (2002) in connection with the discovery of the exoplanet system GJ 876 . This resonance will be investigated in distinct physical models in the forthcoming Sections to test how the semimajor axis separation between the two planets depends on their mass and on the gas density.

\subsection{Shift in the location of the resonance: massive planets}

When two planets are placed in resonance, the apsidal longitude circulation frequency of the inner and outer one, in absence of the disk potential, are determined by the mutual gravitational perturbations. Since they are in resonance, these frequencies cannot be computed with the usual linear secular theory but they are determined by the resonant interactions. When the disk 


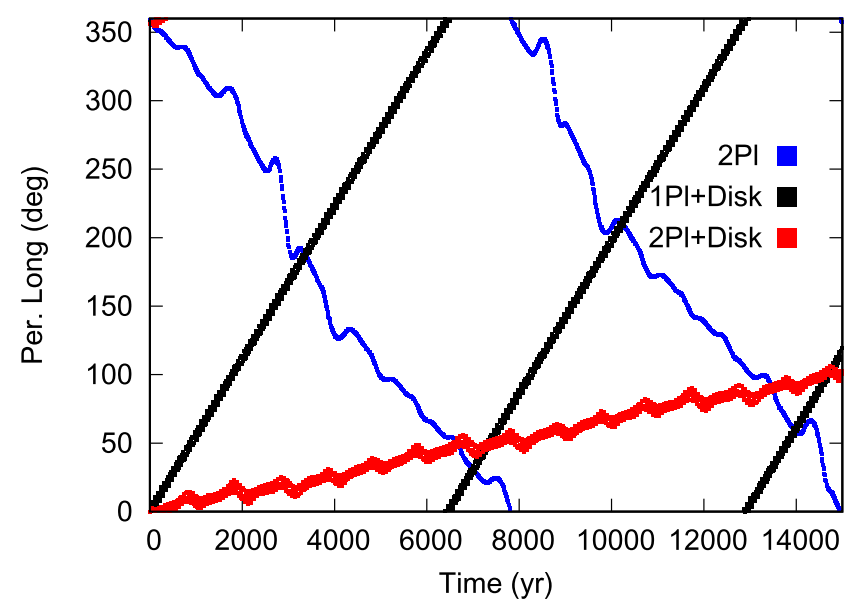

Fig. 1. Evolution with time of the pericenter longitude $\varpi$ of the inner more massive planet as a function of time in three different models, all with a gap in the disk. The blue line shows the evolution of $\varpi$ in a pure three-body problem where the mutual gravitational perturbations of the planets determine the retrograde apsidal longitude circulation of the inner one. The black line illustrates the circulation of the inner planet $\varpi$ affected by the disk potential only (the second planet is not included) which is positive, as expected (Fontana \& Marzari 2016). The full model where both the planets and the disk potential are considered is shown by the red line evolving with a positive frequency because of the dominating effect of the disk potential.

potential is switched on, it acts on both planets affecting the circulation of the apsidal longitude of each body by different amounts since the planets orbit at different locations with respect to the disk or, when present, to the disk gap. This leads to a shift in the resonance location which depends on the contribution of the disk potential to the apsidal circulation of the two bodies. In most cases, the two planets have also a common average rate of apsidal precession which will depend on the disk potential acting on both planets. In this case, the scenario appears even more complex since the disk potential affects the couple of bodies locked in resonance. In Fig. 1 the precession of the apsidal longitude of the inner planet (the more massive) is shown in three different scenarios. In the first a pure three-body problem is numerically integrated and only the gravitational perturbations of the planets are taken into account. In the second the disk potential is applied to both planets assuming they create a common gap in the disk. Finally, we integrate the orbit of the inner planet alone assuming the same potential of the disk as in the previous run (including then the same gap) to evaluate the effect of the disk potential on a single body. Figure 1 clearly shows that the negative circulation frequency of $\varpi$ in the pure three-body problem becomes positive when the gravitational perturbation of the disk is included and this forces an inward shift of the outer planet to maintain the resonance lock.

This behavior affects all the resonant systems, and we expect an inward shift of the outer planet location for all the resonant systems, which depends on the strength of the disk gravity. In Fig. 3 the distribution of the initial semimajor axis of the outer planet for all the sampled resonant systems is illustrated. Three different cases are compared: a reference case where $\Sigma=0$ (pure three-body problem), and two models with the surface density set to match $1 \mathrm{MMSN}$ and $5 \mathrm{MMSN}$, respectively. Each curve shown in Fig. 3 is the outcome of a fit to the histogram of the number of cases versus the initial semimajor axis of the outer planet. The values of the frequencies of the histogram have been normalized so that the underlying area is equal to 1 . A least
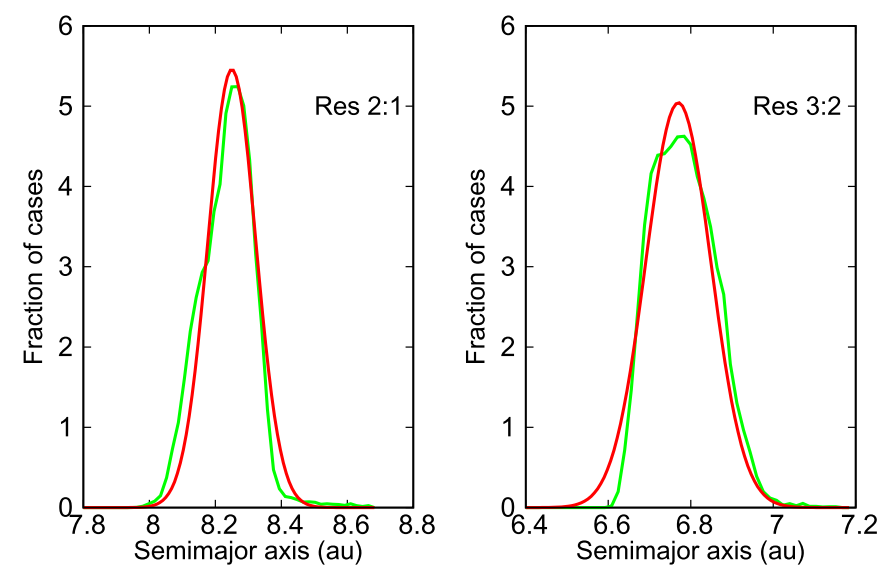

Fig. 2. Gaussian fits to the distribution in semimajor axis of the outer planet in models where the potential of the disk is neglected (pure threebody problem). On the left panel the fit is shown for the $2: 1$ resonance, while, on the right panel, the same fit is shown for the 3:2 resonance.

square fit to the histogram curve is performed with a Gaussian function:

$f(a)=\frac{1}{\sigma \sqrt{2 \pi}} \mathrm{e}^{-\frac{1}{2}\left(\frac{(a-\bar{a})}{\sigma}\right)^{2}}$,

where $\bar{a}$ is the value of the semimajor axis at the center of the resonance and $\sigma$ estimates the dispersion around the center. In Fig. 2 the Gaussian fit is shown in the pure three-body model for both the 2:1 and 3:2 resonances showing a very good match between the analytical curve and the numerical density function.

The Gaussian fit, normalized (in area) to one, is further multiplied by a coefficient $f$ given by the ratio between the total number of cases when the disk potential is included, divided by the total number of cases in the pure three-body problem. The semimajor axis range of the outer planet, randomly explored while seeking for resonant systems, is the same in all three cases $\left(\Sigma_{0}=0, \Sigma_{0}=1 \cdot \Sigma_{\mathrm{MMSN}}\right.$ and $\left.\Sigma_{0}=5 \cdot \Sigma_{\mathrm{MMSN}}\right)$. Therefore, by counting the number of resonant systems found during the random exploration it is possible to estimate the size of the resonant region and the efficiency of trapping in the three different cases. If we denote the total number of resonant systems found at the end of the random search as $n_{0}$ when $\Sigma=0$, the pure three-body case, then the size of the resonant region for $\Sigma_{0}=1 \cdot \Sigma_{\mathrm{MMSN}}$ is reduced by a factor $n_{1} / n_{0}$ where $n_{1}$ is the final number of resonant cases when $\Sigma_{0}=1 \cdot \Sigma_{\text {MMSN }}$. In the same way, the size of the resonant region is reduced by $n_{5} / n_{0}$, where $n_{5}$ is the final number of resonant cases, when $\Sigma=\Sigma_{5 \text { MMSN }}$.

For higher values of the disk surface density $\Sigma$, the center of the resonant region is shifted inwards by an increasing amount. This is due to the increase of the circulation frequency of the planet apsidal precession forced by the disk gravity. To maintain the libration of $\sigma_{1}$ and/or $\sigma_{2}$, the outer planet approaches the inner one increasing its mean anomaly frequency. In most of our models the planets are also in apsidal resonance and the frequency of the apsidal longitude of the outer planet matches that of the inner one. As a consequence, the secular system responds in a unitary way to the disk perturbations.

There is also a shrinking of the volume in the phase space where stable resonant systems (at east over $1 \times 10^{5}$ yr) can be found for increasing $\Sigma$. This reduction is $98 \%$ for $\Sigma=\Sigma_{1 \mathrm{MMSN}}$ and $33 \%$ for $\Sigma=\Sigma_{5 \text { MMSN }}$. The disk potential, when strong enough, is able to destabilize a significant fraction of potential resonant systems reducing the number of possible stable 


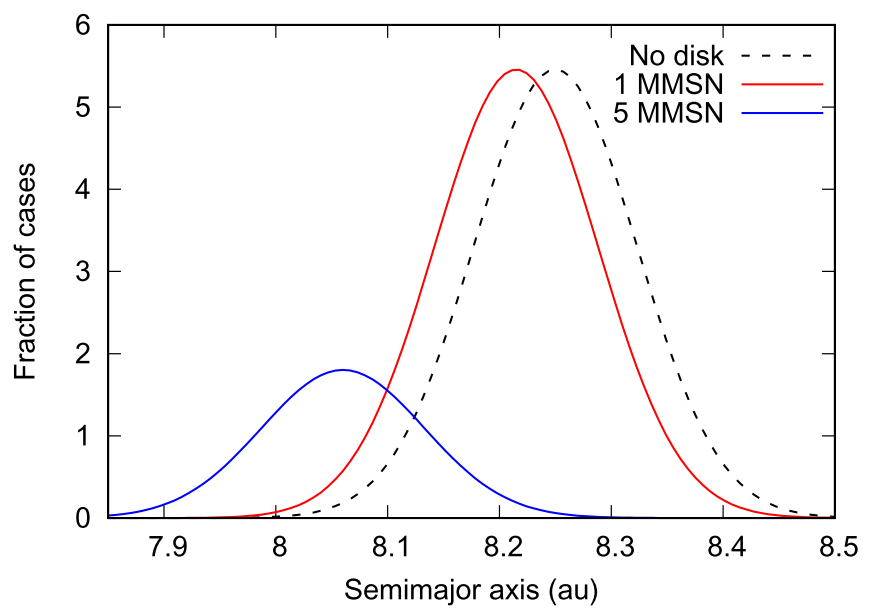

Fig. 3. Distribution of the initial semimajor axes of the outer planet of a massive pair $\left(m_{1}=1 M_{\mathrm{J}}, m_{2}=1 M_{\mathrm{S}}\right)$ trapped in a $2: 1$ resonance for different values of the disk gas density $\Sigma$. For higher densities the resonant region is shifted inwards. The volume of the phase space is also reduced and the size of the resonance region shrinks. This is revealed by the smaller area of the Gaussian curve fitting the histogram.

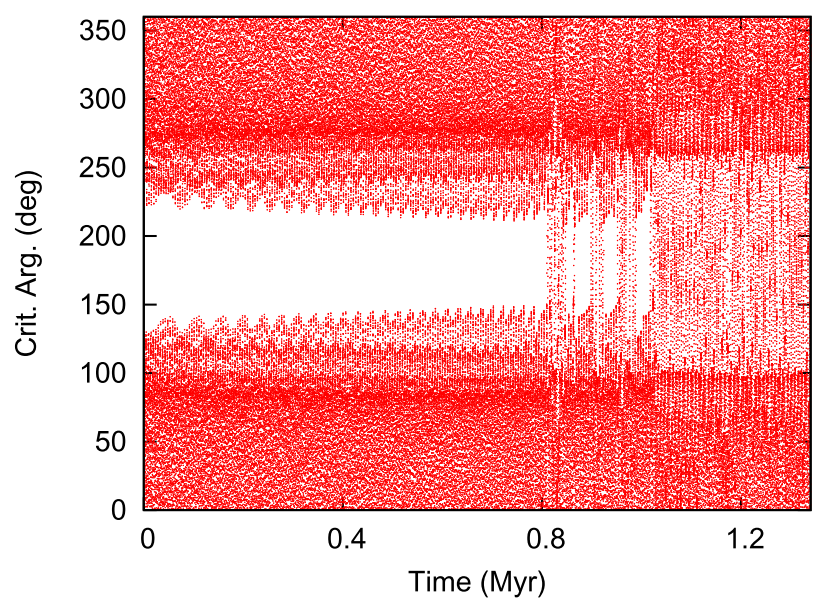

Fig. 4. Time evolution of the $\sigma_{1}$ critical argument of the 2:1 resonance during the dissipation of the disk. The libration slowly turns into circulation (for both $\sigma_{1}$ and $\sigma_{2}$ ) as the density of the gas declines leading to the break up of the resonance. The initial value of $\Sigma$ is equal to $\Sigma_{1 \mathrm{MMSN}}$.

cases. The shift in the apsidal longitude circulation frequency pushes the planets closer to each other increasing their mutual interaction and causing a weakening of the resonance lock.

\subsection{Resonance break up due to disk dissipation}

During the gas dissipation due to photo-evaporation, viscous accretion, and stellar winds, the resonance lock can be broken. In the models where we adopt an exponentially declining $\Sigma$, some systems break out of the resonance and both critical arguments circulate from then on. An example is shown in Fig. 4 where the resonance breakup occurs after about $0.8 \mathrm{Myr}$ with an exponential dissipation coefficient $\tau=5 \times 10^{5} \mathrm{yr}$.

By performing a statistical exploration, through integration of the same systems with and without dissipation, we can estimate the fraction of systems potentially trapped in resonance that are out of it once the disk dissipates. About $27 \%$ of the system initially in resonance is found in a circulating state after a timescale equal to $2 \tau$, confirming that the disk dissipation may

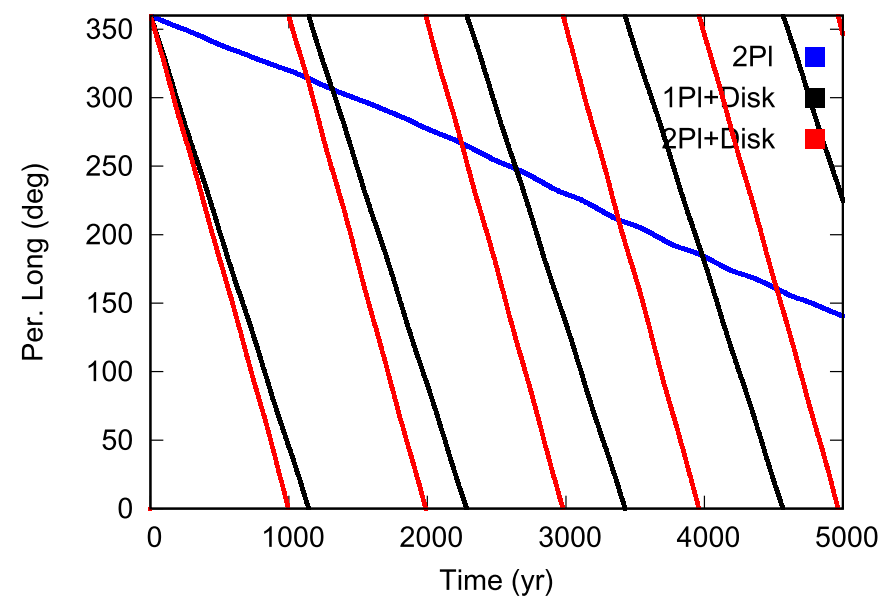

Fig. 5. Evolution with time of the pericenter longitude $\varpi$ in three different models as in Fig. 1. In this case the planets have five and three Earth masses, respectively, and the inner one has a semimajor axis of $1 \mathrm{au}$. The disk potential causes a negative precession which adds up to that due to the mutual gravitational perturbations of the planets. As a consequence, the circulation rate of $\varpi$ is negative in the full model with two planets and the disk potential (red line), contrary to what happens for massive planets within a disk gap. It is also faster compared to that observed in a pure three-body problem (blue line) and for a single planet (black line).

break up the resonance leaving the planets close to it. When the density of the disk is increased to $\Sigma=\Sigma_{5 \text { MMSN }}$ the fraction of cases surviving the disk dissipation drops to $50 \%$. If we neglect all other effects related to the planet-disk interaction and we focus on the disk potential and its effects on the apsidal precession, we find that for higher disk densities the dissipation prevents the evolution from being adiabatic and the system is more easily destabilized.

\subsection{Shift in the location of the resonance: super-Earths}

Here we consider two planets whose masses are five and three times the Earth mass, respectively, with the more massive planet in the inner orbit. They are supposed to be trapped in resonance during their type-I migration or formation process, like insideout growth. Their masses are not large enough to open a gap in the disk and for this reason we adopt for the disk potential Eq. (4) with both planets embedded in the disk. The precession rate of the apsidal longitude due to the disk potential on a single planet is negative (black line in Fig. 5) and concordant with the circulation induced by the resonant interaction (blue line). As a consequence, the circulation rate in the full model including the two planets and the disk (red line in Fig. 5) is faster compared to that of a single planet at 1 au and to that forced by the resonance. This behavior is opposite to that of two massive planets carving a common gap in the disk where the circulation caused by the disk potential is opposite and dominant with respect to the negative one driven by the resonance. Therefore, it is expected that the shifting in the resonance location for super-Earths is outwards rather than inwards as in the case of massive planets.

This is indeed observed in Fig. 6 where we show the distribution of the resonant cases when the semimajor axis of the inner planet is 1 and 5 au, respectively. The shift is outwards in both cases, as expected, but there is a significantly lower reduction of the resonance efficiency when $\Sigma=\Sigma_{5 \text { MMSN }}$ for $a_{1}=1 \mathrm{au}$. The possible reason for this different behavior is that the contribution to the total gravitational potential of the inner part of the disk is 

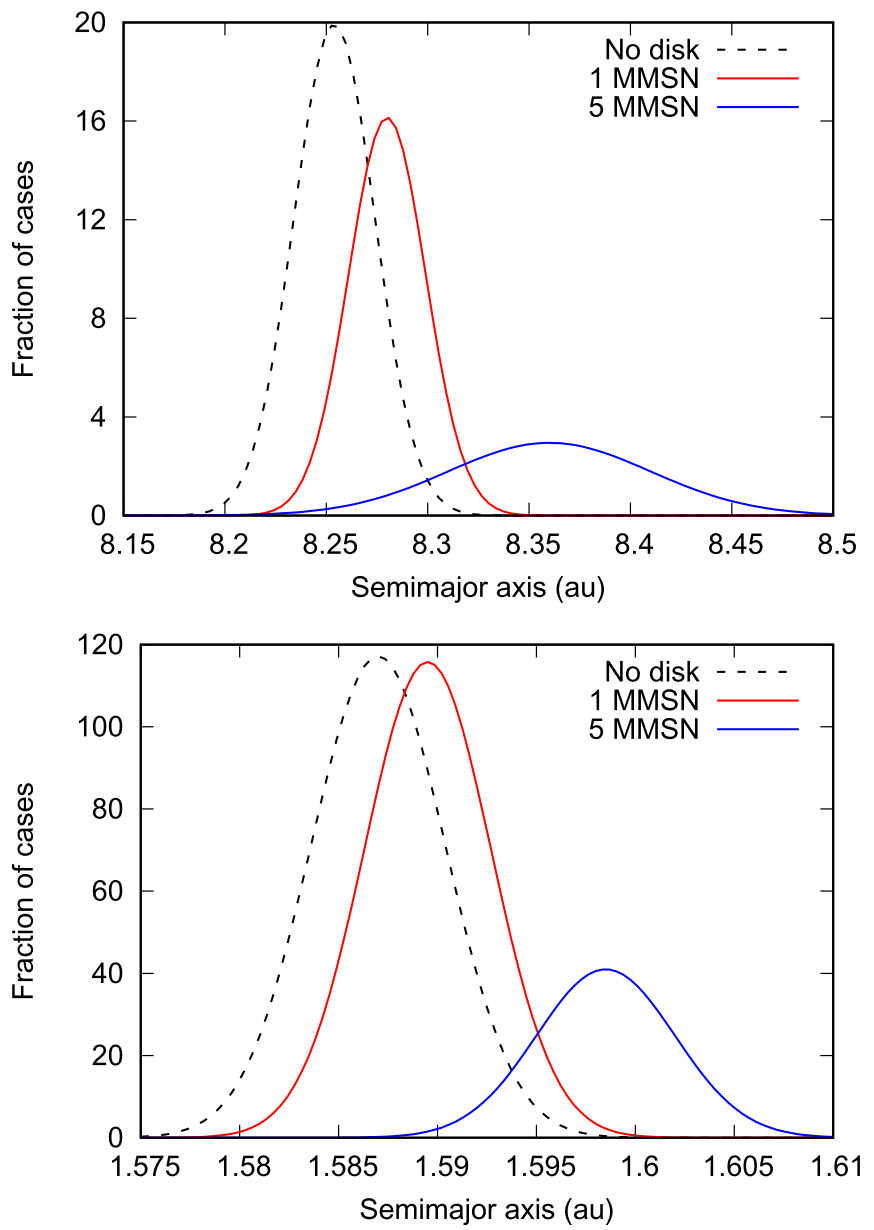

Fig. 6. Distribution of the initial semimajor axes of the outer planet in a system composed of two super-Earths in a 2:1 resonance for different values of the disk gas density. For higher densities the resonant region is shifted inwards. The upper panel models a pair of planets with the inner one at $5 \mathrm{au}$, while the lower panel models the case where the inner planet is at 1 au.

more destabilizing for large densities. As a consequence, since in the "close" case the amount of gas mass in between the inner planet and the star is small and significantly less compared to the "far" case, the resonance is more stable even in the case of high gas density.

\section{The 3:2 resonance}

For Jupiter-size planets, the shift in the resonance location appears less marked for the 3:2 resonance, while, on the other side, the shrink in the resonant region appears more consistent. This is in agreement with the hypothesis that the forced proximity of the planets induced by the shift in the apsidal circulation decreases the stability of the resonant lock. The number of resonant configurations is decreased by about $90 \%$ in the case where the disk density is set to $5 \mathrm{MMSN}$ in comparison to the pure N-body case (Fig. 7). The 3:2 resonance appears more sensitive to the dissipation of the disk gas and about $40 \%$ of the systems are destabilized and escape from resonance before the end of the simulation for $\Sigma=\Sigma_{1 \mathrm{MMSN}}$. The intrinsic weakness of the $3: 2$ resonance to external perturbations, compared to the $2: 1$, suggests that less systems should be found trapped in it but rather are found close to it.

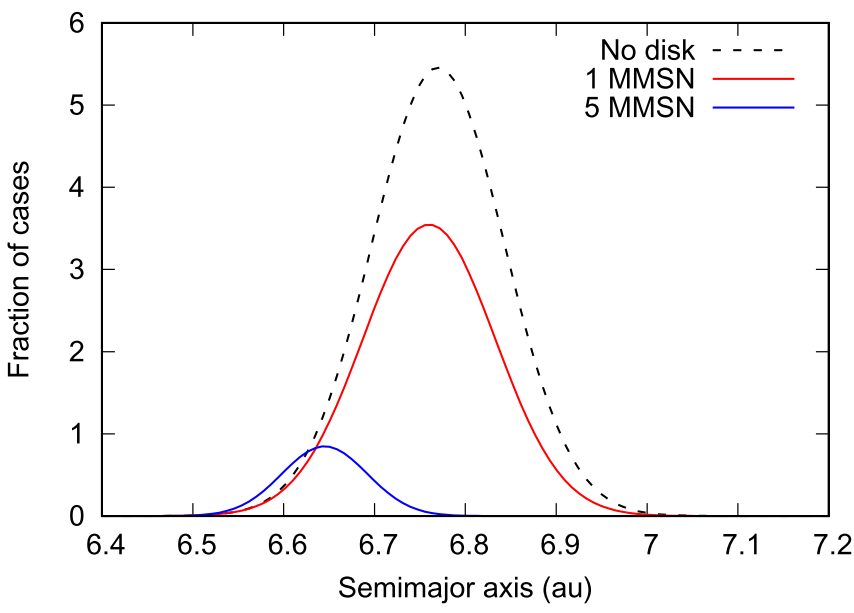

Fig. 7. Distribution of the initial semimajor axes of massive planets in the $3: 2$ resonance for different values of the disk gas density.

When super-Earths are considered (Fig. 8), the shift in the resonant location is present in both the far $\left(a_{1}=5 \mathrm{au}\right)$ and close $\left(a_{1}=1 \mathrm{au}\right)$ configurations, but the resonance efficiency is not significantly decreased in either case. There is a more marked decrease in the "far" case, possibly for the same reasons already outlined at the end of Sect. 5.3 related to the reduced contribution of the inner part of the disk that may be responsible for most of the instability at higher densities. However, there is potentially a dependence on the mass of the planets since the instability for super-Earth pairs is significantly less compared to giant planet pairs. This may be related to the direction of the shift that is outwards for massive planets and inwards for super-Earths.

The dissipation of the disk affects also the stability of superEarths and in the model with a declining gas density the number of cases which are destabilized is about $25 \%$ when $\Sigma=\Sigma_{1 \text { MMSN }}$. In Fig. 9 the behavior of the critical argument of the resonance is shown for two super-Earths when $a_{1}=1 \mathrm{au}$. As in Fig. 4, the critical argument progressively increases its libration amplitude until circulation takes place. In some cases, immediately after the resonance breakup, the planets, before the end of the simulation, have mutual close encounters and undergo a period of chaotic behavior.

\section{Discussion and conclusions}

The coexistence of planets and the gaseous disk suggests interesting dynamical scenarios where the gravitational forces between the planets are perturbed by the disk gravity. Exchange of angular momentum may occur due to the planet perturbations on the disk leading to different types of migration depending on the mass of the planets. An additional effect is the change in the secular frequencies of a planet due to the disk gravitational force. This last effect is of no relevance if the interest is focused on the migration speed of the planets, however it may be of importance when dealing with two planets in resonance. The shift in the secular frequencies leads to a change in the resonance location of the outer planet once the semimajor axis of the inner one is fixed. This shift in the resonance location may be inwards or outwards depending on the mass of the planets and the formation (or not) of a common gap.

In this paper the amount of the resonance shift is numerically estimated by a massive exploration of the phase space around the 2:1 and 3:2 resonances. The shift depends on the disk density and 

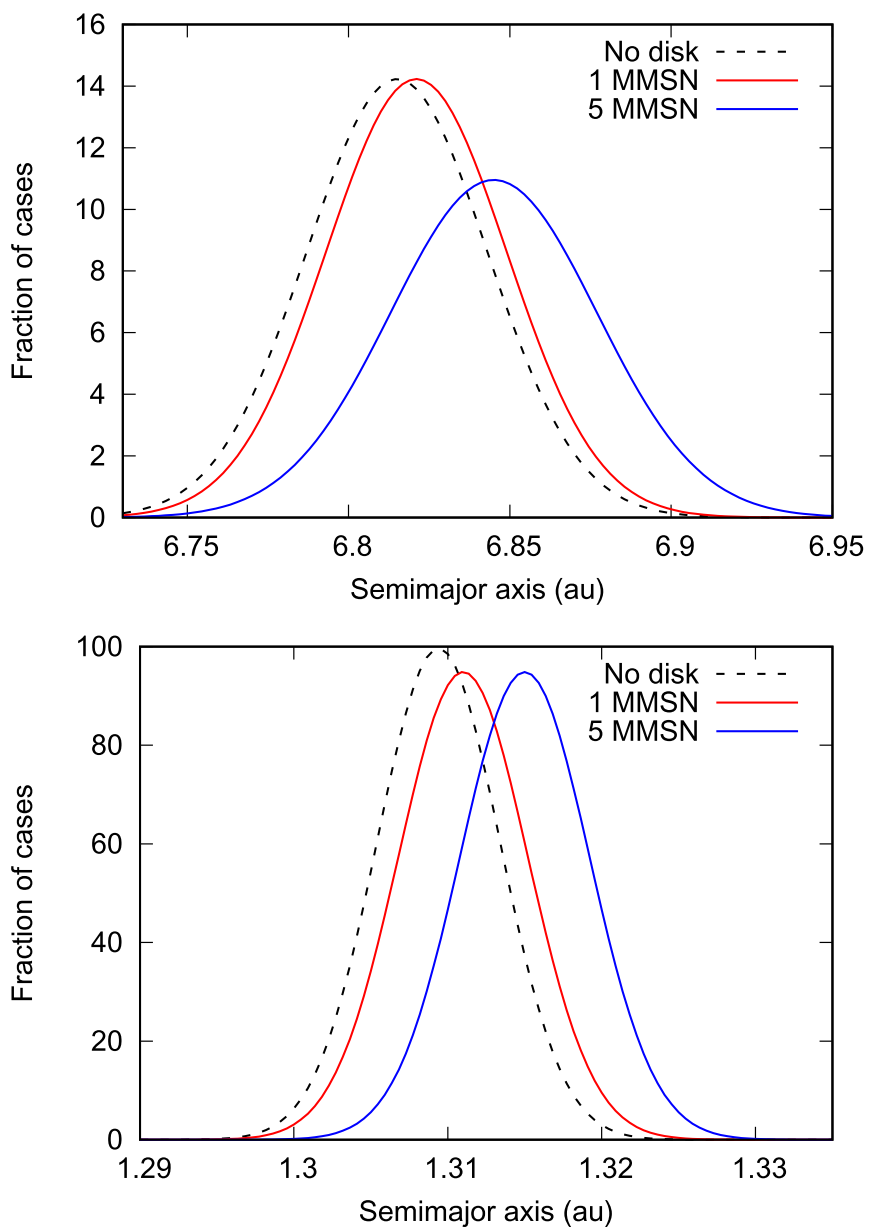

Fig. 8. Distribution of the initial semimajor axes of the outer planet in a system composed of two super-Earths in the 3:2 resonance for different values of the disk gas density. The upper panel models a pair of planets with the inner one at $5 \mathrm{au}$, while the lower panel models the case where the inner planet is at $1 \mathrm{au}$.

on the mass of the planets. The focus here is on Jupiter-Saturnlike couples and super-Earths. When the disk is very massive ( 5 MMSN) the shift is consistent and the stability of the resonance is jeopardized by the strong attraction of the disk. The percentage of systems surviving in resonance decreases significantly as a function of the disk density for both resonances. This effect is less relevant for super-Earths, in particular when a "close" configuration is considered where the inner planet is located at 1 au from the star. In this case the perturbative effects of the inner regions of the disk are reduced and a higher stability is granted.

It is reasonable to expect that during the evolution of the system the disk dissipates due to different mechanisms, such as, photo-evaporation and viscous evolution, among others. In this scenario, the resonance lock may be broken and the planets are left either in a stable configuration close to the resonance or they evolve chaotically during mutual close encounters until a planet is ejected from the system or a collision occurs. This may explain why a significant fraction of planets are found close to a resonance but not exactly in it.

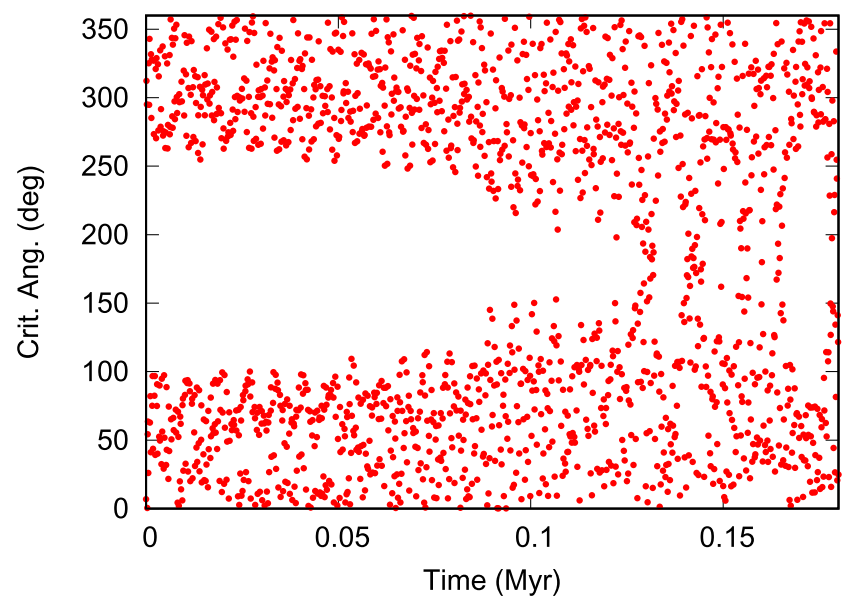

Fig. 9. Time evolution of the $\sigma_{1}$ critical argument of the 3:2 resonance during the dissipation of the disk for the super-Earth case. The libration width grows with time and finally it turns into circulation (for both $\sigma_{1}$ and $\sigma_{2}$ ) when the density of the gas declines. This leads to the break up of the resonance and a period of chaotic evolution characterized by mutual close encounters. The initial value of $\Sigma$ is equal to $\Sigma_{1 \mathrm{MMSN}}$.

Acknowledgments. I thank A. Fontana and an anonymous referee for their useful comments and suggestions.

\section{References}

Beaugé, C. \& Michtchenko, T. A. 2003, MNRAS, 341, 760

Beaugé, C., Michtchenko, T. A., \& Ferraz-Mello, S. 2006, MNRAS, 365, 1160 Binney, J., \& Tremaine, S. 2008, Galactic Dynamics, 2nd edn. (New Jersey: Princeton University Press)

Callegari, Jr., N., Michtchenko, T. A. \& Ferraz-Mello, S. 2004, Celest. Mech. Dyn. Astron., 89, 201

Charpinet, S., Fontaine, G., Brassard, P., et al. 2011, Nature, 480, 496

Chatterjee, S., \& Tan, J. C. 2014, ApJ, 780, 53

Chiang, E. \& Laughlin, G. 2013, MNRAS, 431, 3444

Chiang, E., \& Youdin, A. N. 2010, Ann. Rev. Earth Planet. Sci., 38, 493

Crida, A., Sándor, Z., \& Kley, W. 2008, A\&A, 483, 325

D’Angelo, G., \& Marzari, F. 2012, ApJ, 757, 50

Fabrycky, D. C., \& Kepler Science Team. 2012, in AAS/Division of Dynamical Astronomy Meeting, 43, 1.03

Fontana, A., \& Marzari, F. 2016, A\&A, 589, A133

Hansen, B. M. S., \& Murray, N. 2013, ApJ, 775, 53

Isella, A., Guidi, G., Testi, L., et al. 2016, Phys. Rev. Lett., 117, 251101

Lee, M. H., \& Peale, S. J. 2002, ApJ, 567, 596

Masset, F. 2000, A\&AS, 141, 165

Masset, F., \& Snellgrove, M. 2001, MNRAS, 320, L55

Mestel, L. 1963, MNRAS, 126, 553

Mills, S. M., Fabrycky, D. C., Migaszewski, C., et al. 2016, Nature, 533, 509

Moorhead, A. V., \& Adams, F. C. 2005, Icarus, 178, 517

Raymond, S. N., \& Cossou, C. 2014, MNRAS, 440, L11

Rein, H., Papaloizou, J. C. B., \& Kley, W. 2010, A\&A, 510, A4

Silsbee, K., \& Rafikov, R. R. 2015, ApJ, 798, 71

Steffen, J. H., Fabrycky, D. C., Agol, E., et al. 2013, MNRAS, 428, 1077

Tamayo, D., Triaud, A. H. M. J., Menou, K., \& Rein, H. 2015, ApJ, 805, 15

Thommes, E. W. 2005, ApJ, 626, 1033

Walsh, K. J., Morbidelli, A., Raymond, S. N., O’Brien, D. P., \& Mandell, A. M. 2011, Nature, 475, 206

Ward, W. R. 1981, Icarus, 47, 234

Wright, J. T., Veras, D., Ford, E. B., et al. 2011, ApJ, 730, 93 